\title{
CIANAMIDA HIDROGENADA, THIDIAZURON E ÓLEO MINERAL NA QUEBRA DA DORMÊNCIA E NA PRODUÇÃO DO PESSEGUEIRO cv. CHIRIPÁ ${ }^{1}$
}

\author{
JOSÉ LUIS DA SILVA NUNES² ${ }^{2}$ GILMAR ARDUINO BETTIO MARODIN³ ; IVAR ANTÔNIO SARTORI ${ }^{4}$
}

\begin{abstract}
RESUMO - Este trabalho foi realizado no ano de 1999, na Estação Experimental Agronômica da Universidade Federal do Rio Grande do Sul, localizada no município de Eldorado do Sul, Estado do Rio Grande do Sul, latitude $30^{\circ} 05^{\prime} 52^{\prime \prime}$ S, longitude $51^{\circ} 39^{\prime} 08^{\prime \prime} \mathrm{W}$ e altitude de 46 metros. Foram testados os efeitos da Cianamida Hidrogenada (C.H.) e Óleo Mineral (O.M.) em mistura, Óleo Mineral (O.M.) e Thidiazuron (TDZ) com Óleo Mineral (O.M.), sobre brotação, frutificação, produção e antecipação de colheita. Os tratamentos foram aplicados quando as plantas estavam no estádio fenológico A. Foram testados os seguintes tratamentos: C.H. 0,5\%, 1,0\% e 1,5\%+ O.M 1,0\%, O.M. 1,0\% e 2,0\%, TDZ 200 e 400 ppm + O.M. 2,0\% e testemunha (sem pulverização). Os tratamentos não anteciparam a brotação das gemas vegetativas. O tratamento C.H. 1,5\% com O.M. 1,0\% proporcionou produção suficiente para viabilizar o cultivo do "Chiripá" em condições de inverno ameno, mas não houve diferenças significativas no peso médio dos frutos. O TDZ, nas dosagens testadas, não se mostrou eficiente na quebra de dormência desta cultivar. Não houve efeito na antecipação da colheita.
\end{abstract}

Termos para indexação: Prunus persica, pêssego, quebra de dormência.

\section{HYDROGEN CYANAMIDE, THIDIAZURON AND MINERAL OIL ON BREAKING THE DORMANCY AND PRODUCTION OF PEACHES cv. CHIRIPÁ}

SUMMARY - The present work was conducted in 1999, at the Estação Experimental Agronômica of the Universidade Federal do Rio Grande do Sul, located in the county of Eldorado do Sul, Rio Grande do Sul, latitude 30 $05^{\prime} 52^{\prime \prime}$, longitude 51 $39^{\prime}$ ' $08^{\prime \prime}$ W and altitude at 46 meters. Were tested the effects of the Hydrogen Cyanamide (H.C.) and Mineral Oil (M.O.) in mixture, Mineral Oil (M.O.) and Thidiazuron (TDZ) with Mineral Oil (M.O.), on bud break, yield and anticipation of the crop. Trees were spayde at bud stage A. The following treatments were tested: H.C. $0,5 \%, 1,0 \%$ and $1,5 \%+$ M.O. 1,0\%, M.O. 1, $0 \%$ and 2,0\%, TDZ 200 and 400 ppm + M.O. 2,0\% and control (without any spray). The treatments did not result in anticipation of the bud burst. The treatment H.C. 1,5\% and M.O. 1,0\% provided enough yield to make possible the cultivation of "Chiripá" in mild winters conditions, but there was not significant difference in fruit weight. TDZ, at the tested concentrations, was not efficient to break the dormancy of this cultivar. No effect or crop anticipation was observed.

Index terms: Prunus persica, peach, dormancy breaking.

\section{INTRODUÇÃO}

O Rio Grande do Sul é o maior produtor nacional de pêssegos com 45,22\% da produção (Mondin, 1999). Dentre as cultivares de maior exigência de frio, destaca-se a Chiripá, como a mais importante na serra gaúcha, tendo sido criada pela EMBRAPA de Pelotas em 1975. É uma planta com exigência de frio entre 400 e 500 horas, vigor mediano e frutos destinados ao consumo in natura, de forma redondo-ovalada, com sutura desenvolvida e pequena ponta, de tamanho médio a grande, película creme, com $30 \%$ de vermelho, polpa branca, firme, vermelha junto ao caroço e livre deste, sabor doce e baixa acidez. A maturação ocorre na primeira semana de janeiro e a colheita, no início da segunda semana do mesmo mês. É uma cultivar muito suscetível à podridão-parda - Monilinia fruticola (Wint) Honey, (Medeiros et al., 1998).

A quebra de dormência das plantas caducifólias envolve fatores internos, como balanço dos promotores e inibidores de crescimento, e fatores externos, como temperatura, fotoperíodo e radiação solar, entre outros. Dos fatores externos, o que mais se destaca é a temperatura no inverno, sendo que, quando as plantas são cultivadas em regiões com insuficiências de frio hibernal, apresentam sintomas de falta de adaptação, como atraso e maior duração do período de floração e abertura de menor número de gemas floríferas e vegetativas, resultando em redução na produção, com frutos desuniformes e de baixa qualidade (Marodin et al., 1992).

Para completar sua formação, as gemas floríferas e vegetativas do pessegueiro devem atravessar um período de repouso, cuja medida está relacionada com temperaturas abaixo de $7,2^{\circ} \mathrm{C}$. Constatou-se, porém, que temperaturas acima de $7,2^{\circ} \mathrm{C}$ também têm influência, principalmente em cultivares de menor exigência. A necessidade, para pessegueiro, foi satisfeita com temperaturas médias de $9,7^{\circ} \mathrm{C}$ (Marodin et al., 1991).

1 (Trabalho 192/2000). Recebido: 01/09/2000. Aceito para publicação: 10/07/2001.

1 Eng. ${ }^{\circ}$ Agr. ${ }^{\circ}$, Mestrando, Faculdade de Agronomia da UFRGS, Caixa Postal 776, 91501-970, Porto Alegre - RS, e-mail tobnunes@ig.com.br.

2 Eng. ${ }^{\circ}$ Agr. ${ }^{\circ}$, M.Sc., Dr., Prof. Faculdade de Agronomia da UFRGS, CP 776, 91501-970, Porto Alegre - RS, e-mail marodin@vortex.ufrgs.br.

3 Eng. $^{\circ}$ Agr. ${ }^{\circ}$, M.Sc., Doutorando, Faculdade de Agronomia da UFRGS, e-mail ivar@vortex.ufrgs.br. 
Em muitos anos, não ocorre a superação, efetivamente, da dormência das plantas pela insuficiente acumulação de frio. Nestes casos, a utilização de produtos químicos é prática comum na viabilização dos cultivos de frutíferas de clima temperado. Associações de óleo mineral e sais de dinitro foram as combinações mais utilizadas na quebra da dormência das frutíferas temperadas no Brasil (Petri, 1986). Porém, o uso dos sais de dinitro foi proibido, fazendo com que a melhor opção para a quebra de dormência passasse a ser a utilização de Cianamida Hidrogenada (C.H.), Óleo Mineral (O.M.) e nitrato de potássio (Araújo et al., 1991). A utilização de Thidiazuron (TDZ) em macieira (Fortes \& Araújo, 1991) e C.H. e O.M. em pessegueiro da cultivar Chiripá (Marodin et al., 1989), apresentaram resultados promissores na quebra da dormência.

Uma série de fatores, como concentração dos produtos, épocas de aplicação, estádio das gemas, volume da solução aplicado por planta, somatório das horas de frio acumuladas pela planta e do ingrediente ativo, levam à quebra de dormência, sendo necessários trabalhos para identificar tais fatores para cada local e cultivar (Francisconi et al., 1992). O objetivo principal deste trabalho foi avaliar o efeito de produtos químicos na quebra da dormência, na produção e na antecipação da colheita da cultivar Chiripá.

\section{MATERIAL E MÉTODOS}

O experimento foi conduzido no ano de 1999, em pomar de pessegueiro da cultivar Chiripá, com 8 anos de idade, localizado no Setor de Horticultura e Silvicultura da Estação Experimental Agronômica da Universidade Federal do Rio Grande do Sul (EEA/UFRGS), situada no município de Eldorado do Sul, Estado do Rio Grande do Sul, latitude $30^{\circ} 05^{\prime}$ '52" S, Longitude 51³9'08" W e altitude de 46 metros.

$\mathrm{Na}$ Tabela 3, estão registrados os dados meteorológicos na EEA/UFRGS, situada no município de Eldorado do Sul, de janeiro a dezembro de 1999. A partir de dados coletados em termoigrógrafo, verificou-se que o número de horas de frio iguais ou inferiores a $10^{\circ} \mathrm{C}$ em maio, junho, julho, agosto e setembro foi de 56; 87; $98 ; 83$ e 38 , respectivamente, totalizando 362 horas.

O delineamento experimental utilizado foi o de blocos ao acaso, com 4 repetições, uma planta por parcela e 8 tratamentos. Todos os tratos culturais necessários foram realizados segundo recomendações técnicas difundidas na região, como a poda realizada em 4 de agosto de 1999. O tipo de poda usado foi o de desponte e desbaste dos ramos do ano, onde os ramos mais vigorosos e verticais foram retirados e, nos débeis e laterais, foi feita a eliminação dos direcionados para o centro e dos excedentes de fora da planta, conservando-se os laterais sem despontes. Foram amostrados quatro ramos periféricos por planta nos quatro quadrantes da planta que não sofreram poda de desponte. Nestes ramos, foram avaliados, através da contagem das gemas, as seguintes variáveis: percentual de gemas vegetativas e floríferas brotadas, além das observações fenológicas.

No dia 06 de agosto, com as gemas em estádio fenológico A, foram aplicados os seguintes tratamentos:1) C.H. 0,5\% + O.M. 1,0\%; 2) C.H. 1,0\% + O.M. 1,0\%; 3) C.H. 1,5\% + O.M. 1,0\%; 4) O.M. 1,0\%; 5) O.M. 2,0\%; 6) TDZ 200 ppm + O.M. 2,0\%; 7) TDZ 400 ppm + O.M. 2,0\%; 8) Testemunha.
Como fontes de ingrediente ativo, foram utilizados os produtos comerciais "Dormex" (49\% de C.H.), óleo mineral "Assist" (756 gramas por litro de óleo emulsionável) e "Dropp" (50\% de TDZ). A aplicação foi feita com pulverizador costal manual, com gasto médio de 2,5 litros de calda por planta (10 litros em 4 repetições). O dia da aplicação (06 de agosto) estava com neblina e sem vento, com temperatura média do ar de $20^{\circ} \mathrm{C}$ e $90 \%$ de umidade relativa do ar às 9 horas da manhã.

Foram obtidos dados em duas colheitas (03 e 07 de janeiro de 2000), onde se avaliaram os parâmetros de percentual de colheita em cada data (transformação: arco-seno Öx), produção em peso, número de frutos e peso médio dos frutos através de análise de variância a 5\% de probabilidade e, em caso de significância, procederam-se as comparações entre médias pelo teste de Duncan, ao mesmo nível de probabilidade.

\section{RESULTADOS E DISCUSSÃO}

A percentagem de gemas vegetativas brotadas após 12 dias da aplicação foi baixa em todos os tratamentos, não havendo diferenças significativas em relação à testemunha (Tabela 1), o que evidenciou que os tratamentos não tiveram a ação esperada no período. Isto se deve, provavelmente, à pequena acumulação de horas de frio do período, que não supriram as necessidades para a superação da dormência das gemas vegetativas. Houve uma tendência de o tratamento C.H. 1,5\% + O.M. 1,0\% apresentar maior percentual de gemas brotadas, fato que se refletiu no maior número de frutos e maior produção por planta (Tabela 2).

Verificou-se, também, que os tratamentos não anteciparam a colheita em relação à testemunha (Tabela 1), em desacordo com o trabalho de Marodin et al. (1991), no qual houve antecipação de uma semana na colheita, quando as plantas de pessegueiro "Premier" foram tratadas com doses de C.H. de $0,5 \%$, associado ou não a O.M., e de Fortes \& Araújo (1991), em que o tratamento com TDZ em doses de 20 a 320 ppm, associado a O.M., antecipou a brotação, floração e colheita de maçã cv. Golden Delicius. Isto se deve, provavelmente, ao fato de que, no ano de 1999, as exigências de horas de frio não foram compensadas pelos produtos, o que levou à não-superação natural da dormência das gemas. Além disto, os meses de agosto e setembro tiveram temperaturas bastante baixas (Tabela 3), quando eram necessárias temperaturas mais elevadas para que houvesse uma brotação mais uniforme das gemas.

Para os dados de produção (Tabela 2), constatou-se que o melhor tratamento foi C.H. $1,5 \%+$ O.M. $1,0 \%$, com rendimento comparável à melhor média de produção da região, mas sem diferir do O.M. 2\% em número e peso de frutos e TDZ 400ppm + O.M. $2 \%$ em peso de fruto por planta. A causa provável foi a maior capacidade de produção de fotoassimilados, devido ao melhor enfolhamento que este tratamento proporcionou, e ao maior número de frutos por planta, devido a uma provável menor queda natural dos frutinhos, em conformidade com os resultados obtidos por Marodin et al. (1989) com a mesma cultivar que, utilizando C.H. acima de $0,5 \%$, associado ou não a O.M., obteve menor queda natural dos frutos, mas que não foi medido neste trabalho. Com relação ao peso médio dos frutos, verificou-se que não houve diferença significativa entre os tratamentos e a testemunha, mostrando que a baixa quantidade de frutos de alguns tratamentos 
TABELA 1 - Percentagem de gemas vegetativas brotadas aos 12 dias após aplicação e percentual da colheita, em duas datas, em pessegueiro "Chiripá". Eldorado do Sul-RS, 1999.

\begin{tabular}{|c|c|c|c|}
\hline & Gemas vegetativas - & Colh & ita \\
\hline Tratam entos & $\begin{array}{c}\text { brotadas } 12 \text { dias a ós } \\
(\%)\end{array}$ & $\begin{array}{c}03 / 01 \\
(\%)\end{array}$ & $\begin{array}{c}07 / 01 \\
(\%)\end{array}$ \\
\hline $1-\mathrm{C} \cdot \mathrm{H} \cdot 0,5 \%+O \cdot M \cdot 1,0 \%$ & $26,33 \mathrm{ab}$ & $52,81^{*}$ & $47,19 *$ \\
\hline $2-\mathrm{C} \cdot \mathrm{H} \cdot 1,0 \%+\mathrm{O} \cdot \mathrm{M} \cdot 1,0 \%$ & 32,47 a b & 78,60 & 21,40 \\
\hline $3-\mathrm{C} \cdot \mathrm{H} \cdot 1,5 \%+\mathrm{O} \cdot \mathrm{M} \cdot 1,0 \%$ & $35,50 \mathrm{a}$ & 42,78 & 57,22 \\
\hline $4-\mathrm{O} . \mathrm{M} \cdot 1,0 \%$ & 27,32 ab & 46,10 & 53,90 \\
\hline $5-O . M \cdot 2,0 \%$ & 23,48 a b & 35,05 & 64,95 \\
\hline $6-\mathrm{TDZ} 200 \mathrm{ppm}+\mathrm{O} \cdot \mathrm{M} \cdot 2,0 \%$ & 24,92 ab & 56,92 & 43,08 \\
\hline $\begin{array}{l}\text { 7- TDZ400ppm +O.M. } 2,0 \% \\
8 \text { - Testemunha }\end{array}$ & $\begin{array}{l}18,26 \text { b } \\
24,37 \text { a b }\end{array}$ & $\begin{array}{l}42,68 \\
62,42\end{array}$ & $\begin{array}{l}57,32 \\
3758\end{array}$ \\
\hline C.V. (\%) & 36,00 & $-1-1-1$ & - \\
\hline
\end{tabular}

Médias seguidas da mesma letra não diferem significativamente, pelo teste de Duncan, a 5\%.

* Não significativo.

TABELA 2 - Número e peso de frutos colhidos por planta e peso médio dos frutos em pessegueiro "Chiripá". Eldorado do Sul-RS, 1999.

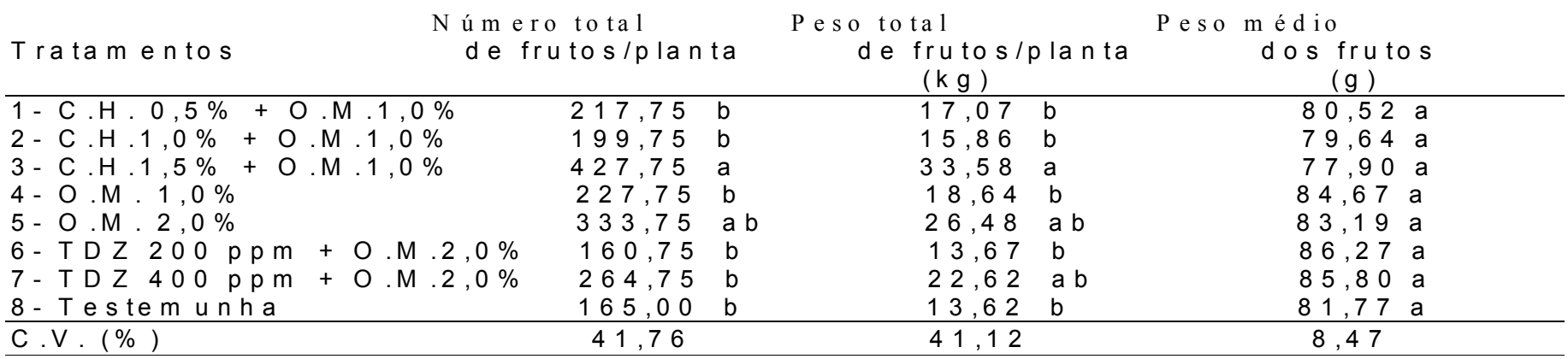

Médias seguidas da mesma letra não diferem significativamente, pelo teste de Duncan, a $5 \%$.

TABELA 3 - Dados climáticos obtidos na Estação Agrometeorológica da EEA -UFRGS, localizada no município de Eldorado do Sul-RS, ano de 1999.

\begin{tabular}{|c|c|c|c|c|c|c|c|}
\hline $\mathrm{M}$ ê $\mathrm{s}$ & M á $x$ im & $\begin{array}{l}\text { T e m peratura } \\
\text { a } \quad M \text { édia }\end{array}$ & M ín im a & $\begin{array}{c}\text { P re c ip ita çã o } \\
(\mathrm{m} \mathrm{m})\end{array}$ & $\begin{array}{l}\cup R \\
(\%)\end{array}$ & $\begin{array}{l}\mathrm{H} \text { oras de } \\
<10^{\circ} \mathrm{C}\end{array}$ & $\begin{array}{l}\text { F rio } \\
<7^{\circ}(\end{array}$ \\
\hline $\mathrm{J}$ a $\mathrm{n}$ & 30,9 & 24,1 & 18,5 & 59,9 & 71 & - & - \\
\hline $\mathrm{F}$ e $\mathrm{v}$ & 29,5 & 23,1 & 17,6 & 103,1 & 75 & - & - \\
\hline$M$ a $r$ & 32,0 & 24,6 & 18,9 & 75,4 & 71 & - & - \\
\hline$A b r$ & 23,5 & 18,1 & 13,3 & 147,8 & 78 & 2 & - \\
\hline$M$ a io & 21,3 & 14,9 & 8,7 & 147,2 & 76 & 49 & 7 \\
\hline $\mathrm{J} u \mathrm{n}$ & 18,5 & 12,7 & 7,6 & 183,0 & 81 & 61 & 26 \\
\hline J u I & 18,4 & 13,0 & 8,1 & 189,5 & 82 & 70 & 28 \\
\hline A go & 21,5 & 14,7 & 8,0 & 62,1 & 80 & 51 & 32 \\
\hline$S e t$ & 224 & 162 & 99 & 930 & 81 & 35 & 3 \\
\hline O u t & 22,7 & 17,6 & 12,6 & 133,4 & 75 & - & - \\
\hline $\mathrm{N} O \mathrm{~V}$ & 26,0 & 19,5 & 12,4 & 115,4 & 71 & 3 & - \\
\hline$D$ e z & 29,0 & 22,5 & 16,7 & 62,0 & 72 & - & - \\
\hline Tota I & $-\ldots$ & $-\ldots$ & 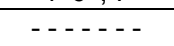 & 1366,8 & $\ldots-\cdots$ & 271 & 96 \\
\hline
\end{tabular}

não resultou em frutos de maior tamanho, provavelmente, pelo baixo enfolhamento e menor produção de fotoassimilados. Os tratamentos com O.M. 2,0\% e TDZ 400 ppm com O.M. 2,0\% não diferiram estatisticamente do melhor tratamento.

Se o somatório de horas de frio fosse efetivo para a superação da dormência e houvesse temperaturas mais elevadas nos meses de brotação, provavelmente, o uso dos tratamentos químicos traria um efeito de antecipação de colheita, como ocorreu no trabalho conduzido com a cultivar Premier por Marodin et al. (1991), que obtiveram antecipação de uma semana em relação à testemunha, nos tratamentos com C.H. $0,5 \%$.

\section{CONCLUSÕES}

1- Os tratamentos não anteciparam a brotação das gemas vegetativas, em comparação com a testemunha.

2- O melhor tratamento: C.H. 1,5\% com O.M. 1,0\% proporcionou produção suficiente para viabilizar o cultivo do "Chiripá" em condições de inverno ameno, mas não diferiu estatisticamente de O.M. 2\% E TDZ com O.M. 2\%.

3- Não houve efeito de antecipação de colheita por parte dos tratamentos em relação à testemunha.

\section{REFERÊNCIAS BIBLIOGRÁFICAS}

ARAÚJO, M. M.; FORTES, G. R. de L.; SANTOS FILHO, B. Thidiazuron: uma alternativa para superar a dormência de gemas de macieira (Malus domestica, Borkh). Revista Brasileira de Fruticultura, Cruz das Almas, v.223, n.3, p. 249 - 253.1991. 
FORTES, G. R. de L.; ARAÚJO M. M. Thidiazuron: uma citocinina potente para superar a dormência de gemas de macieira (Malus domestica, Borkh, L.). II - Concentração do produto. In: CONGRESSO BRASILEIRO DE FISIOLOGIA VEGETAL, 3 , Vicosa, S B FV/Universidade Federal de Viçosa,1991.p.61.

FRANCISCONI, A. H. D; MARODIN, G. A. B.;;; GALLOIS, E. S. P. Efeito do Óleo Mineral, Cianamida Hidrogenada e Thidiazuron na quebra de dormência e produção de Pereira (Pyrus comunis, L.) cv. Packham's Triumph. Revista Brasileira de Fruticultura, Cruz das Almas, v.14, n. 1, p. 161 - 166, 1992.

MARODIN, G. A. B.; LUCHESE, O. A.; MANFROI, V. A Cianamida Hidrogenada e Óleo Mineral na quebra de dormência e produção do pessegueiro "Premier". Revista Brasileira de Fruticultura, Cruz das Almas, v.13, n.3, p.165-171, 1991.

MARODIN, G. A. B.; FRANCISCONI, A. H. D.; GALLOIS, E. S. P. Efeito de produtos químicos na quebra de dormência e produção de Pereira (Pyrus communis, L.) cv Packham's Triumph.
Revista Brasileira de Fruticultura, Cruz das Almas, v.14, n.1, p.155 - 160, 1992.

MARODIN, G. A. B.; LUCHESE, O. A.; MANFROI, V.; GERHARDT, I. R.; AMARO, S.S. A Cianamida hidrogenada e Óleo Mineral na quebra da dormência e produção do pessegueiro "Chiripá". In: CONGRESSO BRASILEIRO DE FRUTICULTURA, 10, 1989. Fortaleza, CE, Resumos... Sociedade Brasileira de Fruticultura, 1989. 56 p.

MEDEIROS, C. A. B.; RASEIRA, M. do C. B. A cultura do pessegueiro. Brasília: EMBRAPA - SPI; Pelotas: EMBRAPA CPACT, 1998.351 p.

MONDIN, V. P. Frutas de clima temperado, Videira: EPAGRI, 1999. $18 \mathrm{p}$.

PETRI, J. L. Dormência da macieira. In:. EMPASC. Manual da Cultura da Macieira. Florianópolis, SC, 1986. Cap.7, p.163-201. 